tNSAIDs had the most patients with emergency department visits $(20.8 \%$ vs. $16.7 \%$ in both COX-2s and oral tNSAIDs), and COX-2 had the most patients with inpatient visits (18.1\% vs. $15.4 \%$ for topical tNSAIDs and $11.8 \%$ for oral tNSAIDs). Oral tNSAIDs had the lowest total all-cause cost $(\$ 6,504)$, and the topical tNSAIDs cohort had the highest costs $(\$ 8,455)$, but fairly comparable with COX-2s $(\$ 8,289)$. During follow-up, oral tNSAIDs patients stayed mostly on oral tNSAIDs as less than $15 \%$ of oral tNSAIDs patients later had a prescription for COX-2s or topical tNSAIDs. $37 \%$ of COX-2 patients and $56 \%$ of topical tNSAIDs patients later took oral tNSAIDs. Topical tNSAIDs patients had an average of 184.4 days of supply for topical tNSAIDs yet also extensively used oral NSAIDs during follow-up (average days of supply for oral tNSAIDs was 315.5 days and for COX-2s was 383.5 days)

Conclusion: This study suggests that patients with more complex comorbidity profiles, including higher rates of adverse effects, often start pharmacological treatment with topical tNSAIDs. However, patients who start treatment with topical tNSAIDs switch to other types of NSAIDs; oral tNSAIDs were the most frequently prescribed treatment across the cohorts. Thus, despite the safety concerns with oral tNSAIDs and COX-2s, patients are still placed on these treatments to manage their OA pain. There is a need for new innovative treatments as there is currently a lack of other options.

Disclosure of Interests: Stuart Silverman Consultant of: Stuart Silverman is a paid consultant to Pfizer and Eli Lilly and Company in connection with this study, Patricia Schepman Shareholder of: Patricia Schepman is an employee of Pfizer with stock and/or stock options, Employee of: Pfizer, James B Rice Consultant of: Brad Rice is an employee of Analysis Group, who were paid consultants to Pfizer and Eli Lilly and Company for this study, Craig Beck Shareholder of: Craig Beck is an employee of Pfizer with stock and/or stock options, Employee of: Pfizer, Alan White Consultant of: Alan White is an employee of Analysis Group, who were paid consultants to Pfizer and Eli Lilly and Company for this study, Sheena Thakkar Shareholder of: Sheena Thakkar is an employee of Pfizer with stock and/or stock options, Employee of: Pfizer, Michaela Johnson Consultant of: Michaela Johnson is an employee of Analysis Group, who were paid consultants to Pfizer and Eli Lilly and Company for this study, Rebecca Robinson Shareholder of: Rebecca Robinson is an employee and minor stockholder of Eli Lilly and Company, Employee of: Eli Lilly and Company, Birol Emir Shareholder of: Birol Emir is an employee of Pfizer with stock and/or stock options, Employee of: Pfizer DOI: 10.1136/annrheumdis-2021-eular.2175

\section{POS0284 CLINICAL AND MRI COMPARISON OF ECCENTRIC VERSUS CONCENTRIC REHABILITATION IN SYMPTOMATIC KNEE OSTEOARTHRITIS: A PROSPECTIVE RANDOMIZED STUDY}

M. C. Trojani ${ }^{1}$, D. Bendahan ${ }^{2}$, F. Chorin ${ }^{3}$, P. Gerus ${ }^{4}$, V. Breuil ${ }^{5}$, S. Guis ${ }^{6}$, C. Roux ${ }^{7} .{ }^{1}$ Université cote d'azur, Rheumatology, Nice, France; ${ }^{2}$ Aix Marseille University, CNRS, Centre de Résonance Magnétique Biologique et Médicale (CRMBM), UMR 7339, Marseille, France; ${ }^{3}$ Université Cote d'Azur, LAMHESS laboratory, NICE, France; ${ }^{4}$ Université Cote d'Azur, LAMHESS laboratory, Nice, France; ${ }^{5}$ Université Cote d'Azur, Rheumatology, Nice, France; ${ }^{6}$ AixMarseille University, 13009, Marseille, France, CRMBM UMR CNRS 7339, Marseille, France: ${ }^{7}$ Université cote d'azur, Rheumatology, Nice, France

Background: Rehabilitation is at the center of the non-medical management of knee osteoarthritis. Concentric muscle strengthening is often preferred, while eccentric contractions play an important role in controlling knee flexion and knee stability and allow the development of a high level of strength with a low energy cost. But few studies have focused on these two technique

Objectives: To explore the effect of a 6-week-exercise program on function, pain and performance level in symptomatic knee osteoarthritis patients

Methods: An analysis was performed of the data from 60 individuals with symptomatic knee osteoarthritis who were included in the EXART study. The EXART study was a prospective, randomized controlled trial which included patients aged 40 to 85 with KL 2 or 3 responding to the American College of Rheumatology criteria. The first group benefitted from a 6 week eccentric rehabilitation program and the second group from a standard 6 week concentric rehabilitation program. The endpoints were the changes from baseline to week 6 in Western Ontario and McMaster Universities Osteoarthritis Index (WOMAC), pain, and performance measured with quadriceps power (PMax) and contraction strength (MMax). MRI muscle analysis was performed before and after physical activity. Results: Among 80 patients screened, 60 were included in the study and randomized. 25/30 subjects finished the program in the concentric group and 28/30 in the eccentric group. The mean population age was $74(+/-8)$, mean IMC 27.2 $(+/-4)$. The baseline total WOMAC mean was $49.2(+/-19)$, VAS pain $5.3(+/-2)$. At 6 weeks, both groups showed a significant improvement compared to baseline for the WOMAC without any difference between the two groups (mean difference evolution concentric $=7.5 \pm 11.9(p=p<0.0001)$; mean difference evolution eccentric $=4.5 \pm 1.9(p<0.0001)$; intergroup analysis $p=0.7)$. Similar significant results were found in the WOMAC function subscale. No difference between the groups appeared in VAS pain evolution $(p=0.7)$. The eccentric group showed a significant improvement in PMax and high speed MMax evolution ( $p=0.001$ and $p=0.002)$. This improvement was not found for the concentric group $(p=0.52$ and $p=0.27$ ). MRI showed a vastus medialis hypertrophy only in the eccentric group $(p=0.002)$. We did not observe any change in the fatty infiltration of the quadriceps on MRI.

Conclusion: Rehabilitation, whether eccentric or concentric, has a beneficial action on function and pain in symptomatic knee osteoarthritis whatever the methods. A gain in muscle performance and vastus medialis volume was found only with the eccentric rehabilitation. This study confirms the importance of any type of rehabilitation in knee osteoarthritis and raises the question of the relation between muscle gain/performance and function or pain

Acknowledgements: We would like to thank all the patients who took part in the study as well as all the members of the Fragile Platform of the Nice University Hospital.

Disclosure of Interests: None declared

DOI: 10.1136/annrheumdis-2021-eular.3164

\section{POS0285 ARE RACIAL DISPARITIES IN REVISION TKA OUTCOMES ASSOCIATED WITH HOSPITAL OR SURGEON VOLUME?}

S. Mirza ${ }^{1}$, S. Goodman ${ }^{2,3}$, Y. Zhang ${ }^{2}$, H. Do ${ }^{2}$, B. Mehta ${ }^{2,3}$, S. Lyman ${ }^{2}$, L. A. Mandl ${ }^{2,3}$, M. Figgie ${ }^{4}$, M. Parks ${ }^{4}$, L. Russell ${ }^{2,3}$, A. Bass ${ }^{2,3}{ }^{1}$ Touro College of Osteopathic Medicine, Medical School, New York, United States of America; ${ }^{2}$ Hospital for Special Surgery, Medicine, New York, United States of America; ${ }^{3}$ Weill Cornell Medical Center, Medicine, New York, United States of America; ${ }^{4}$ Hospital for Special Surgery, Orthopedic Surgery, New York, United States of America

Background: Total knee arthroplasty (TKA) outcomes are linked to surgical volume, ${ }^{1}$ despite the increase in TKA utilization, racial disparities in TKA outcomes persist. Blacks in the US are at a higher risk of aseptic revision of TKA (R-TKA) when compared to Whites, yet the reasons for this are not understood.

Objectives: The objective of this study is to examine the relationship between hospital and surgeon annual TKA volume and R-TKA outcomes by race.

Methods: This is an observational cohort study. New York Statewide Planning and Research Cooperative System data for 2004 - 2013 was used to identify patients who underwent primary TKA. Data through 2015 was used to identify R-TKA within 2 years of the index TKA. Hospital characteristics were obtained from the AHA Annual Survey. Surgeon data was collected from New York State Education Department and New York State Physician Profile. Surgeon annual TKA volume was categorized based on cutoffs established by Wilson et $\mathrm{al}^{1}$ as $</=12,13-59,60-145$ or $>/=146$, and hospital TKA volume as $</=89,90-235,236-644$ and $>/=645$. We calculated the odds of R-TKA in Whites and Blacks separately and generated crude odds ratios (OR) comparing Blacks to Whites to examine trends across volume categories. A multivariable logistic regression model adjusted for known R-TKA risk factors was also performed.

Results: A total of 163,576 patients were included. Mean (SD) age was 66.4 (10.4) years, 107,233 (65.6\%) were female, 124,277 (76.6\%) were White and 15,990 (9.8\%) were Black. 2925 patients underwent aseptic R-TKA. In logistic regression analysis, Blacks had a higher risk of R-TKA (OR 1.42, $95 \% \mathrm{Cl} 1.26$ 1.6) (Table 1). Risk of R-TKA was also higher when surgeon annual volume was $</=12$ (OR $1.5,95 \% \mathrm{Cl} 1.25-1.8$ ) or $13-59$ (OR $1.16,95 \% \mathrm{Cl} 1.04-1.29)$ TKA compared to the highest volume surgeons $(>/=146)$. Patients who had surgery at a hospital with annual volume of 236-634 TKA were less likely to undergo R-TKA compared to the highest volume hospitals $(>/=645)$ (OR $0.88,95 \% \mathrm{C}$ 0.79-0.98). Other risk factors for R-TKA were younger age and worker's compensation, while patients with inflammatory arthritis had a lower risk. Figures $1 \mathrm{~A}$ and $1 \mathrm{~B}$ show the odds of R-TKA in Whites and Blacks, respectively, by hospital and surgeon volume. Figure $1 \mathrm{C}$ shows the crude $\mathrm{OR}$ for Blacks to Whites for each category pair. The OR ranged from 0.9 to 2.5 , with the largest disparity found in patients who have TKA performed by surgeons with 60-145 annual TKA volume at the highest volume hospitals $(>/=645)$.

Conclusion: Patients having TKA by a surgeon performing $<60$ TKA per year have higher risk of R-TKA. Racial disparities in R-TKA risk are highest for TKA by surgeons performing 60-145 TKA per year at hospitals performing $>/=645$ TKA per year. Future studies should examine factors, such as whether trainees are involved the surgery, that may vary based on social determines of health, such as patient race and payor.

\section{REFERENCES:}

[1] Wilson S. et al Meaningful thresholds for the volume-outcome relationship in total knee arthroplasty. Journal of bone and joint surgery. 2016 98:1683 